\title{
Structural view of the regulatory subunit of aspartate kinase from Mycobacterium tuberculosis
}

\author{
Qingzhu Yang ${ }^{1}$, Kun $\mathrm{Yu}^{2}$, Liming $\mathrm{Yan}^{2}$, Yuanyuan $\mathrm{Li}^{1}$, Cheng Chen ${ }^{2}$, Xuemei $\mathrm{Li}^{1 凶}$ \\ ${ }^{1}$ National Laboratory of Biomacromolecules, Institute of Biophysics, Chinese Academy of Sciences, Beijing 100101, China \\ 2 Structural Biology Laboratory, Tsinghua University, Beijing 100084, China \\ \ Correspondence: lixm@sun5.ibp.ac.cn \\ Received August 7, 2011 Accepted September 1, 2011
}

\begin{abstract}
The aspartate kinase (AK) from Mycobacterium tuberculosis (Mtb) catalyzes the biosynthesis of aspartate family amino acids, including lysine, threonine, isoleucine and methionine. We determined the crystal structures of the regulatory subunit of aspartate kinase from Mtb alone (referred to as MtbAK $\beta$ ) and in complex with threonine (referred to as MtbAK $\beta$-Thr) at resolutions of $2.6 \AA$ and $2.0 A$, respectively. MtbAK $\beta$ is composed of two perpendicular non-equivalent ACT domains [aspartate kinase, chorismate mutase, and TyrA (prephenate dehydrogenase)] per monomer. Each ACT domain contains two $\alpha$ helices and four antiparallel $\beta$ strands. The structure of MtbAK $\beta$ shares high similarity with the regulatory subunit of the aspartate kinase from Corynebacterium glutamicum (referred to as CgAK $\beta$ ), suggesting similar regulatory mechanisms. Biochemical assays in our study showed that MtbAK is inhibited by threonine. Based on crystal structure analysis, we discuss the regulatory mechanism of MtbAK.
\end{abstract}

KEYWORDS Mycobacterium tuberculosis, aspartate kinase, crystal structure, $\beta$ subunit

\section{INTRODUCTION}

As one of the most deadly global diseases, tuberculosis (TB) causes about 9 million active infection cases and 2 million casualties every year (Tomioka and Namba, 2006). Although several chemotherapy drugs, including pyrazinamide, ethambutol, isoniazid and rifampicin are clinically effective, the increasing incidences of multidrug-resistant
(MDR) Mycobacterium tuberculosis (Mtb) strains and coinfection of $M$ tb and human immunodeficiency virus (HIV) have made the control of $M$ tb more complicated and challenging and the development of novel therapeutic antiTB drugs in urgent demands (Corbett et al., 2003; Chan and Iseman, 2008).

The biosynthesis pathway of aspartate family amino acids is essential for the development and survival of most bacteria, including Mtb. Functioning in the initial step of this pathway, aspartate kinase (AK) catalyzes the phosphorylation of aspartic acid to 4-phospho-L-aspartate, which is subsequently converted to lysine, threonine, isoleucine and methionine (Chaitanya et al., 2010). One of the intermediate products, diaminopimelic acid, contributes to the cross-linked structure of peptidoglycan, the strength and rigidity of bacterial cell wall (Chaitanya et al., 2010). Experiments using $M$. smegmatis showed that the deletion of the gene coding for AK results in significant cell growth inhibition (Rapaport et al., 1996). A negative regulatory feedback of AK by end products has been reported in Corynebacterium glutamicum (CgAK) and Thermus thermophilus (TtAK) (Cole et al., 1998; Yoshida et al., 2007a; Yoshida et al., 2009). Although no negative feedback regulation has been reported in MtbAK, a similar inhibitory regulation may exist in MtbAK. Based on these critical roles played by AK in amino acid biosynthesis pathway, which is absent in human, AK has been identified as a potential drug target in $M t b$, especially in MDR-Mtb (Cirillo et al., 1994; Chaitanya et al., 2010).

Two types of AKs exist, homo-oligomeric and heterotetrameric. AKI from Arabidopsis thaliana is an example of homo-oligomeric AK (Rognes et al., 1980; Mas-Droux et al., 2006; Schuldt et al., 2011). AKs from M. tuberculosis (Cirillo et al., 1994; Gilker J.M., 1997; Schuldt et al., 2011), C. 
glutamicum (Yoshida et al., 2007a) and T. thermophiles (Nishiyama et al., 1995; Yoshida et al., 2007b) belong to the second type. The hetero-tetrameric $\alpha_{2} \beta_{2}$ AK contains $\alpha$ and $\beta$ subunits, which are encoded by in-frame overlapping genes (Schuldt et al., 2011). The catalytic domain of heterotetrameric $\alpha_{2} \beta_{2}$ AK is composed of the $\mathrm{N}$-terminal region of the $\alpha$ subunit and the regulatory domain comprises the $\beta$ subunit and the $\mathrm{C}$-terminal region of the $\alpha$ subunit (Gilker J.M., 1997; Yoshida et al., 2007a, 2007b; Schuldt et al., 2011). A key feature of the regulatory domain of $A K$ is the conserved ACT domain [aspartate kinase, chorismate mutase, and TyrA (prephenate dehydrogenase)] (Aravind and Koonin, 1999; Chipman and Shaanan, 2001), which comprises a $\beta \alpha \beta \beta \alpha \beta$ fold and is expected to play a regulatory role when binding to small molecules (Grant, 2006). The monofunctional AKs can be divided into three classes depending on the number of ACT domain in the regulatory domain. Class I has two ACT domains per monomer; class II contains two ACT domains per subunit and class III has four ACT domains per monomer. Homo-oligomeric AK belongs to class I or class III, while the hetero-tetrameric AK belongs to class II (Robin et al., 2010). Aspartokinase from M. tuberculosis which has 421 residues is a monofunctional $\alpha_{2} \beta_{2}$-type aspartokinase encoded by a single gene. The a subunit is comprised of residues 1-421. The $\beta$ subunit from $M$. tuberculosis is comprised of residues 250-421. The residues 250-421 of C-terminal region of the $\alpha$ subunit and the $\beta$ subunit make up the regulatory domain. Each subunit contains two ACT domains (Gilker J.M., 1997; Cole et al., 1998; Fleischmann et al., 2002; Schuldt et al., 2011).

Crystal structures of several homo-oligomeric AKs and hetero-tetrameric $\alpha_{2} \beta_{2}$-type AKs have been reported previously, such as the homo-oligomeric $A K$ from $A$. thaliana (PDB code: 2CDQ) (Mas-Droux et al., 2006), the $\beta$ subunit of $\alpha_{2} \beta_{2}$-type AK from C. glutamicum (PDB code: 2DTJ) (Yoshida et al., 2007a), the $\beta$ subunit of $\alpha_{2} \beta_{2}$-type AK from $T$. thermophiles (TtAK $\beta$ ) (PDB code: $2 \mathrm{ZHO}$ and 2DT9) (Yoshida et al., 2009) and the full-length $\alpha_{2} \beta_{2}$-type AK from $C$. glutamicum (PDB code: 3AAW, 3AB2 and 3AB4) (Yoshida et al., 2010). However, no crystal structure of MtbAK has been reported and the regulatory mechanism of MtbAK is poorly understood. In this study, the crystal structures of MtbAK $\beta$ alone (MtbAK $\beta$-free) and in complex with threonine (MtbAK $\beta$ Thr) have been determined at resolutions of $2.6 \AA$ and $2.0 \AA$,

Table 1 Data collection statistics

\begin{tabular}{|c|c|c|}
\hline Parameter & MtbAK $\beta$-free & MtbAK $\beta-T h r$ \\
\hline \multicolumn{3}{|l|}{ Data collection statistics } \\
\hline \multirow[t]{3}{*}{ Cell parameters $(\AA$, deg $)$} & $a=b=64.8 \AA$ & $a=b=62.4 \AA$ \\
\hline & $c=137.0 \AA$ & $c=137.3 \AA$ \\
\hline & $\alpha=\beta=\gamma=90^{\circ}$ & $\alpha=\beta=\gamma=90^{\circ}$ \\
\hline Space group & 141 & 141 \\
\hline Wavelength used $(\AA)$ & 1.0000 & 0.9800 \\
\hline Resolution range $(\AA)$ & $50.00-2.60(2.64-2.60)$ & $50.00-2.00(2.03-2.00)$ \\
\hline No. of all reflections & 42061 & 87898 \\
\hline No. of unique reflections & 8654 & 17555 \\
\hline Completeness (\%) & $99.7(99.0)^{c}$ & $99.7(99.9)^{c}$ \\
\hline $\mathrm{I} / \sigma(\mathrm{I})$ & $21.3(3.1)^{\mathrm{c}}$ & $40.4(3.4)^{\mathrm{c}}$ \\
\hline $\mathrm{R}_{\text {merge }}^{\mathrm{a}}(\%)$ & $13.6(65.6)^{c}$ & $4.3(60.4)^{\mathrm{c}}$ \\
\hline \multicolumn{3}{|l|}{ Refinement statistics } \\
\hline No. of reflections used $(\sigma(F)>0)$ & 8263 & 17540 \\
\hline $\mathrm{R}_{\text {work }}^{\mathrm{b}}(\%)$ & 20.6 & 21.9 \\
\hline $\mathrm{R}_{\text {free }}^{\mathrm{b}}(\%)$ & 26.2 & 27.7 \\
\hline r.m.s.d. bond distance $(\AA)$ & 0.021 & 0.007 \\
\hline r.m.s.d. bond angle (deg) & 1.990 & 1.222 \\
\hline Overall average $B$ factor $\left(\AA^{2}\right)$ & 48.0 & 52.4 \\
\hline \multicolumn{3}{|c|}{ Ramachandran plot (excluding Pro and Gly) } \\
\hline Res. in most favored regions & $129(90.2 \%)^{c}$ & $125(87.4 \%)^{c}$ \\
\hline Res. in additionally allowed regions & $13(9.1 \%)^{c}$ & $14(9.8 \%)^{c}$ \\
\hline Res. in generously allowed regions & $1(0.7 \%)^{c}$ & $1(0.7 \%)^{c}$ \\
\hline
\end{tabular}


respectively. Biochemical assay showed that MtbAK is inhibited by threonine. The regulatory mechanism of MtbAK is further analyzed and discussed, based on the conformational differences between MtbAK-free and MtbAKtheronine.

\section{RESULTS AND DISCUSSION}

\section{Overall structure of MtbAK $\beta$-free and MtbAK $\beta$-Thr}

The crystal structure of the MtbAK $\beta$-free was determined at $2.6 \AA$ by molecular replacement method with a final $R_{\text {work }}$ value of $20.6 \%\left(R_{\text {free }}=26.2 \%\right)$ using the regulatory subunit of $\alpha_{2} \beta_{2}$-type AK from C. glutamicum (PDB code: 2DTJ) (Yoshida et al., 2007a) as a search model. The space group of the crystal is 141 with unit cell parameters of $a=b=64.8 \AA, c=$ $137.0 \AA, \alpha=\beta=\gamma=90^{\circ}$. There is one MtbAK $\beta$ molecule (166 residues) per asymmetric unit with a Matthews coefficient of $3.7 \AA^{3} / \mathrm{Da}$, corresponding to $67 \%$ solvent content (Matthews, 1968). The electron densities of residues 303-304 and 418-421 are not observed on the density map.

The crystal structure of MtbAK $\beta$-Thr was determined at 2.0 $\AA$ resolution with a final $R_{\text {work }}$ value of $21.9 \%\left(R_{\text {free }}=27.7 \%\right)$ by the molecular replacement method using the structure of $M \operatorname{tbAK} \beta$-free as a search model. The space group of the crystal is 141 with unit cell parameters of $a=b=62.4 \AA, c=$ 137.3 $A, \alpha=\beta=y=90^{\circ}$. There is one monomer (166 residues) containing one Thr molecule per asymmetric unit with a Matthews coefficient of $3.3 \AA^{3} / \mathrm{Da}$, corresponding to $63 \%$ solvent content (Matthews, 1968). The electron densities of residues 303-304 and 418-421 are not observed on the map. We summarized final refinement statistics in Table 1.

Each MtbAK $\beta$ contains two perpendicular ACT domains. The N-terminal ACT will be referred to as ACT1 and Cterminal ACT as ACT2. Each ACT is composed of fourstranded $\beta$ sheets and two $\alpha$-helices and are connected by a short loop of two amino acids.

ACT1 and ACT2 are topologically different. ACT1 has a typical $\beta a \beta \beta \alpha \beta$ topology, while ACT2 domain displays a $\beta \beta \alpha \beta \beta \alpha \beta n$ topology, with the first $\beta$ strand in ACT2 in front of ACT1. Similar arrangement of the two ACT domains is also found in AK from C. glutamicum (CgAK) (Yoshida et al., 2007a), AKIII from E. coli (EcAKIII) (Kotaka et al., 2006) and AK from M. jannaschii (MjAK) (Faehnle et al., 2006) and implies a peculiar structural feature of the AK regulatory domain.

The structure of MtbAK $\beta$-Thr contains a monomer and one Thr molecule. The Thr molecule is bound at site 1 in MtbAK $\beta$ while in homo-oligomeric AKs, two inhibitors are bound at one binding pocket (Kotaka et al., 2006; Yoshida et al., 2009).

The main difference between the structures of MtbAK $\beta$ free and MtbAK $\beta$-Thr is the outward shifts of the $\beta$ strands around Thr-binding site in MtbAK $\beta$-Thr (Fig. 5B). The rmsd for<smiles>NC(CC(=O)O)C(=O)O</smiles>

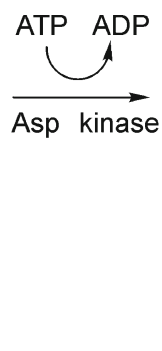<smiles>NC(CC(=O)OP(=O)([O-])[O-])C(=O)O</smiles>

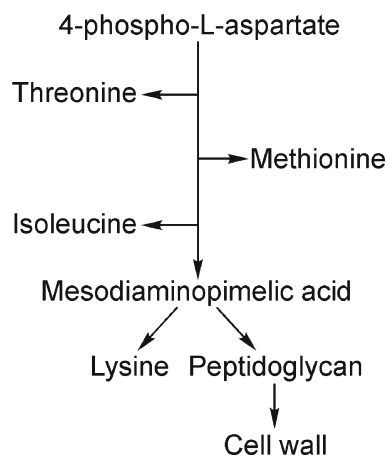

Figure 1. Reaction catalyzed by MtbAK. The first step in the biosynthesis of the aspartate group of amino acids, phosphorylation of aspartic acid, is catalyzed by MtbAK.

Ca between the two structures is about $0.9 \AA$, which will be discussed in detail later.

\section{Comparison with the structures of $\beta$ subunits from other AKs}

MtbAK $\beta$ monomer shares high similarity with $\operatorname{CgAK} \beta$ and TtAK $\beta$, according to superimposition of the structures of MtbAK $\beta$-free, CgAK $\beta$ (PDB code: 2DTJ) and TtAK $\beta$ (PDB code: $2 \mathrm{ZHO}$ ) (Yoshida et al., 2009). The $\beta$ subunits from these three AKs all have two ACT domains with a characteristic $\beta \alpha \beta \beta \alpha \beta$ structure. The high structural similarity between MtbAK $\beta, \operatorname{CgAK} \beta$ and TtAK $\beta$ suggests similar regulatory mechanisms for these regulatory subunits. However, there are two major differences among the three $\beta$ subunits (Fig. 3A and $3 B$ ). First, $\operatorname{CgAK} \beta$ contains an extra $\beta$ strand $\beta 9$ which is not part of the C-terminal ACT2. According to recent studies, this strand is proposed to be crucial for the biological roles of CgAK, which may be different from MtbAK and TtAK (Yoshida et al., 2010). Second, the residues $54-55$ of the $\beta 3-\beta 4$ loop region are disordered in the crystal structure of MtbAK $\beta$, implying high flexibility in this region. Third, about 5 residues at the $\mathrm{N}$-terminus of $\mathrm{CgAK} \beta$ and TtAK $\beta$ are missing due to lack of electron density, while those of MtbAK $\beta$ are observed clearly from electron density map. Although a few other differences are found, they are likely due to the amino acid sequence differences and are not further analyzed. 
A

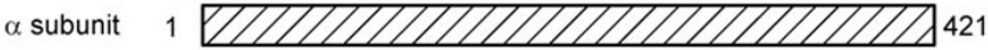

$\beta$ subunit

250 : 1421

B

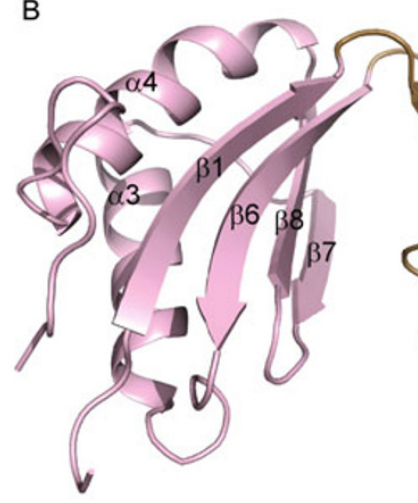

D

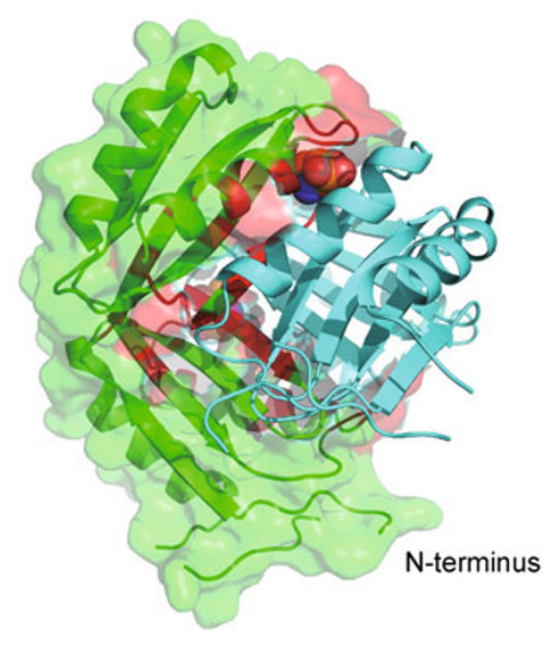

C

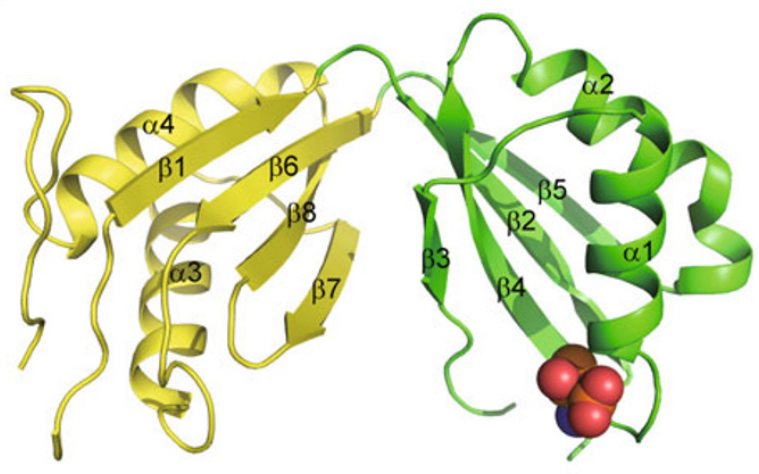

$\mathrm{E}$

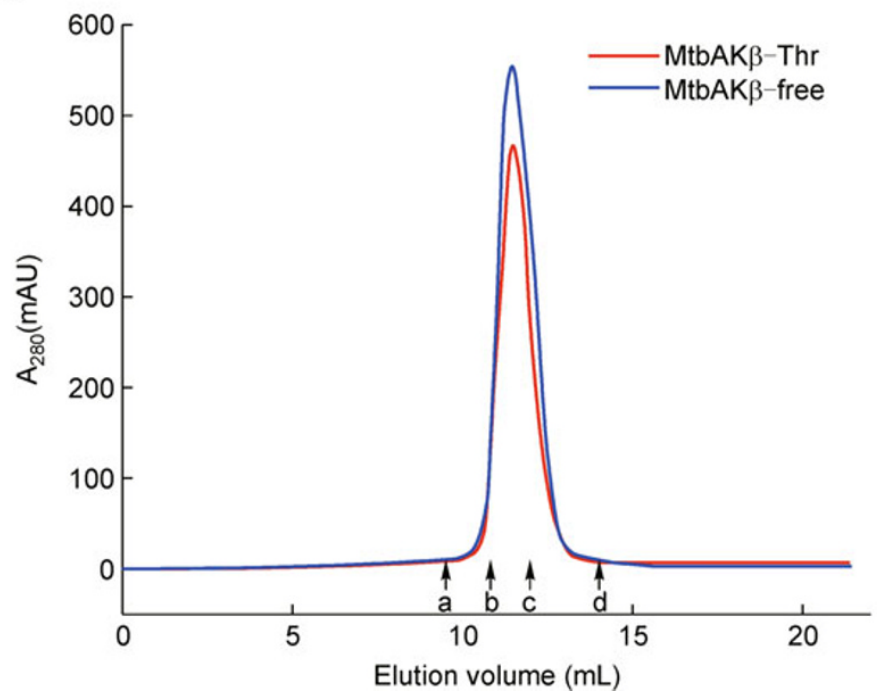

Figure 2. The overlapping genes of MtbAK and crystal structure of MtbAK $\beta$. (A) The $\alpha$ subunit and $\beta$. subunit of MtbAK are shown by striped and dotted bars respectively. The $\beta$ subunit of MtbAK was used for structural study in our work. (B) Overall structure of MtbAK $\beta$-free monomer. The ACT1 and ACT2 domains are indicated by light pink and sand respectively. (C) Overall structure of MtbAK $\beta$-Thr monomer. ACT1 and ACT2 domains are indicated by green and yellow respectively. Bound Thr is shown as orange spheres. (D) The structure of homodimeric MtbAK $\beta$-Thr. The molecules are colored in green and cyan respectively. Bound Thr is shown as orange spheres. The interface, consisting of residues essential for intermolecular contact (distance less than $3.6 \AA$ ), is colored in red. (E) Elution profiles of MtbAK $\beta$ in the presence and absence of $10 \mathrm{mmol} / \mathrm{L}$ Thr. Blue and red lines indicate in the absence and presence of $10 \mathrm{mmol} / \mathrm{L}$ Thr, respectively. Elution volumes for BSA (67.0 kDa), ovalbumin (43.0 kDa), carbonic anhydrase $(29.0 \mathrm{kDa})$ and ribonuclease $\mathrm{A}(13.7 \mathrm{kDa})$ are indicated by a, b, c, and d, respectively. Data were resolved and plotted by MATLAB (http://www.mathworks.com/products/matlab). 
A

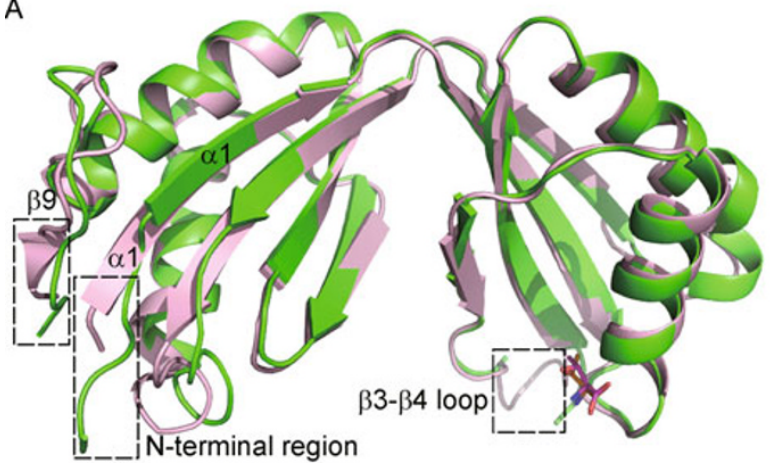



C

M. tuberculosis

M. tuberculosis

C. glutamicum

T. thermophilus

M. tuberculosis

M. tuberculosis C. glutamicum

T. thermophilus

M. tuberculosis

M. tuberculosis C. glutamicum

T. thermophilus



$\alpha 1$

30 elecele

$\underset{50}{\stackrel{33}{\longrightarrow}}$

AAKVF

AAKVFRA A A A INIDM

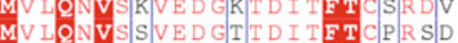

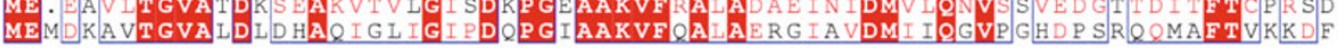



$\alpha 4$

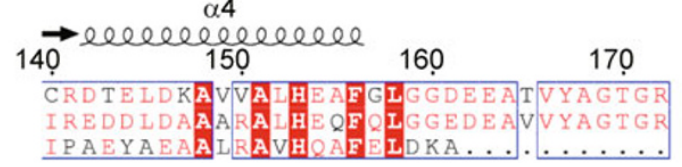

Figure 3. Comparison of the structure of MtbAK $\beta-T h r$ monomer with CgAK $\beta$-Thr and TtAK $\beta$-Thr monomers. (A) Superimposition of MtbAK $\beta$-Thr monomer (green) onto CgAK $\beta$-Thr (PDB code: 2DTJ) monomer (magenta). CgAK $\beta$-Thr contains an extra $\beta$ strand at the $C$ terminus. Bound threonines in MtbAK $\beta$-Thr and CgAK $\beta$-Thr are shown as orange and magenta sticks respectively. (B) Superimposition of MtbAK $\beta$-Thr monomer (green) onto TtAK $\beta$-Thr (PDB code: 2DT9) monomer (lightblue). Bound threonines in MtbAK $\beta$-Thr and TtAK $\beta$-Thr are shown as orange and marine sticks respectively. Positions of difference are marked by dashed boxes. (C) Structure-based sequence comparison of the $\beta$ subunit of aspartate kinases from M. tuberculosis, $C$. glutamicum and $T$. thermophilus. Secondary structure elements of the $\beta$ subunit of aspartokinase from $M$. tuberculosis are shown at top of the alignment.

\section{Analysis of the oligomeric state of MtbAK $\beta$}

TtAK $\beta$ is in equilibrium of monomer and dimer conformations. Thr and/or concentrations of TtAK $\beta$ facilitate the dimerization of TtAK $\beta$ (Yoshida et al., 2009). Similar to TtAK $\beta$, threonine can induce the dimerization of CgAK $\beta$ (Yoshida et al., 2007a). To examine the effect of threonine on MtbAK $\beta$, we investigated the oligomeric state of MtbAK $\beta$ in the presence and absence of Thr by two methods, namely gel filtration chromatography and analytical ultracentrifugation.

Gel filtration chromatography profile showed that MtbAK $\beta$ was exactly eluted at the same volume in the presence and absence of $10 \mathrm{mmol} / \mathrm{L}$ threonine, giving an observed molecular weight of $35.0 \mathrm{kDa}$ (Fig. 2E). These results suggest that addition of Thr does not affect the oligomeric state of MtbAK $\beta$.

Analytical ultracentrifugation confirmed that the estimated molecular mass of MtbAK $\beta$ in the presence of $10 \mathrm{mmol} / \mathrm{L} \mathrm{Thr}$ is $37.0 \mathrm{kDa}$. Considering the mass of a monomer is $20.4 \mathrm{kDa}$, these results show that MtbAK $\beta$ is in a dimer conformation with or without threonine. However, in the case of $\mathrm{CgAK}$, the equilibrium favors the monomer conformation in the absence of threonine, suggesting that the binding affinity between $\beta$ subunits is stronger in MtbAK.

CgAK dissociates into $\alpha$ and $\beta$ subunits during purification in the absence of threonine. The $\beta$ subunit of TtAK is eluted from the column later than the $\alpha$ subunit in the absence of threonine in gel filtration assays (Yoshida et al., 2009). From the binding assay of the $\alpha$ subunits and $\beta$ subunits from 
A

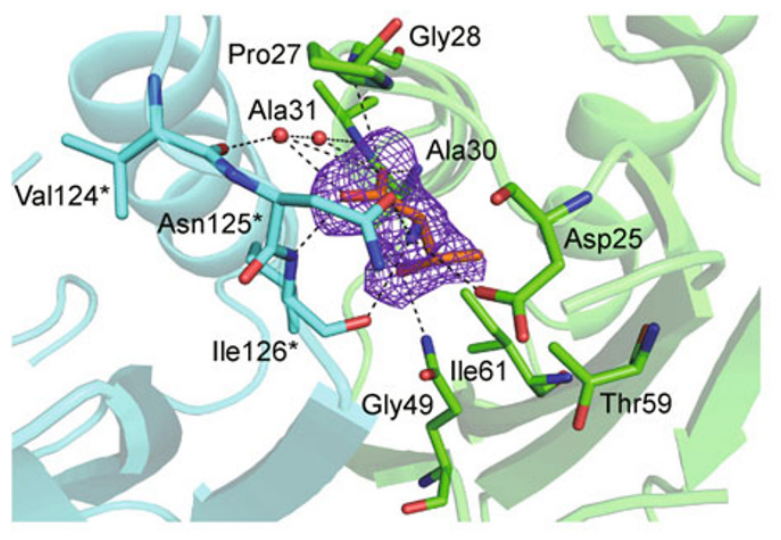

B

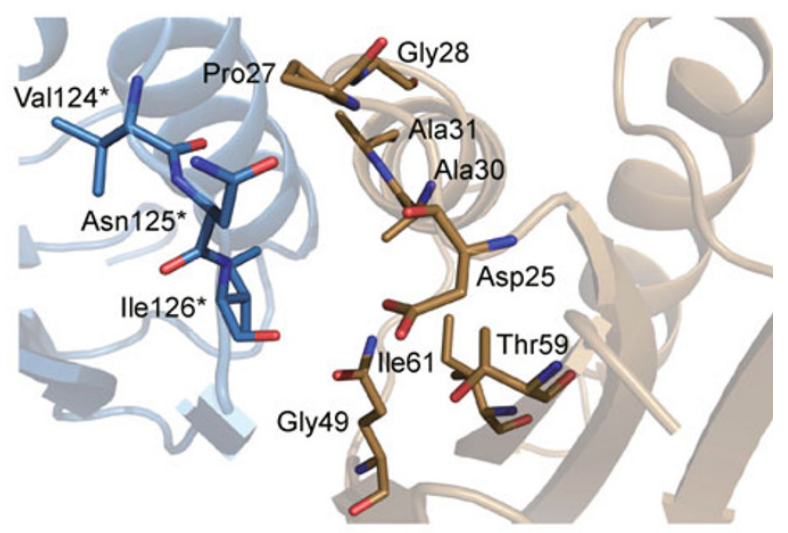

C

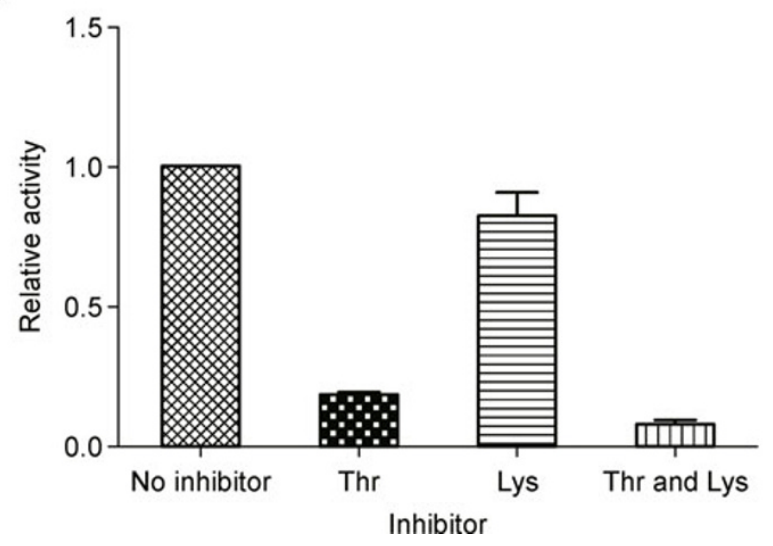

Figure 4. Thr-binding site and the enzyme activity study. (A) Thr-binding site in MtbAK $\beta$-Thr. Green, chain $X$; blue, chain $Y$; orange, Thr. Residues from chain $Y$ are shown with asterisks. Number after the residue represents the residue number from $\beta$ subunit. (B) Vacant Thr-binding site in MtbAKß-free. sand, chain X; skyblue, chain Y; Residues from chain Yare shown with asterisks. Number after the residue represents the residue number from $\beta$ subunit. (C) Inhibition profile of MtbAK by threonine. The histograms represent the mixture with $10 \mathrm{mmol} / \mathrm{L} \mathrm{Thr}, 10 \mathrm{mmol} / \mathrm{L} \mathrm{Lys,} 10 \mathrm{mmol} / \mathrm{L} \mathrm{Thr}$ plus $10 \mathrm{mmol} / \mathrm{L}$ Lys and without inhibitor as control, respectively.

MtbAK, the $\beta$ subunits containing $\mathrm{His}_{6}$ tags at the $\mathrm{C}$ terminus were bound to the $\alpha$ subunits in the absence of threonine, that is to say, MtbAK is purified in the $\alpha_{2} \beta_{2}$ form in the absence of threonine. Moreover, the $\alpha$ subunit and $\beta$ subunit are eluted together from the column in the absence of threonine in gel filtration assays. From these observations, we conclude that the $\alpha$ subunit of MtbAK can interact with the $\beta$ subunit without threonine, and the interaction is stronger than those of TtAK and CgAK.

\section{Dimeric assembly of MtbAK $\beta$}

Crystal structure showed that two molecules of MtbAK $\beta$-free, which are connected by a crystallographic screw fourfold axis, form a sphere-like homodimer with a $1814 \AA^{2}$ intra-dimer interface, while the MtbAK $\beta$-Thr homodimer has an interface of $2065 \AA^{2}$ (Fig. 2D). Multitudinous hydrophobic interactions, hydrogen bonds, salt bridges and van de Waals interactions between the two monomers (Table 2) suggest that the homodimer is stable. MtbAK is a heterotetramer with an $\alpha_{2} \beta_{2}$ configuration (Schuldt et al., 2011). As the $\beta$ subunit of MtbAK is identical to the C-terminal portion of the a subunit, the dimeric structure could characterize the crystal structure of the regulatory region of an $\alpha_{2} \beta_{2}$-heterodimer. This is in accordance with the results of similar $\alpha_{2} \beta_{2}$ AKs such as TtAK and CgAK (Yoshida et al., 2009; Yoshida et al., 2010). It is not clear whether the homodimers described above have functional relevance and more biochemical data are needed to test this hypothesis.

\section{Thr-binding site}

The crystal structure of MtbAK $\beta$-Thr showed that the electron density of a threonine molecule is located at site 1 in the interface between ACT1 domain and ACT2 domain from a different chain and is solvent inaccessible. The structure of 

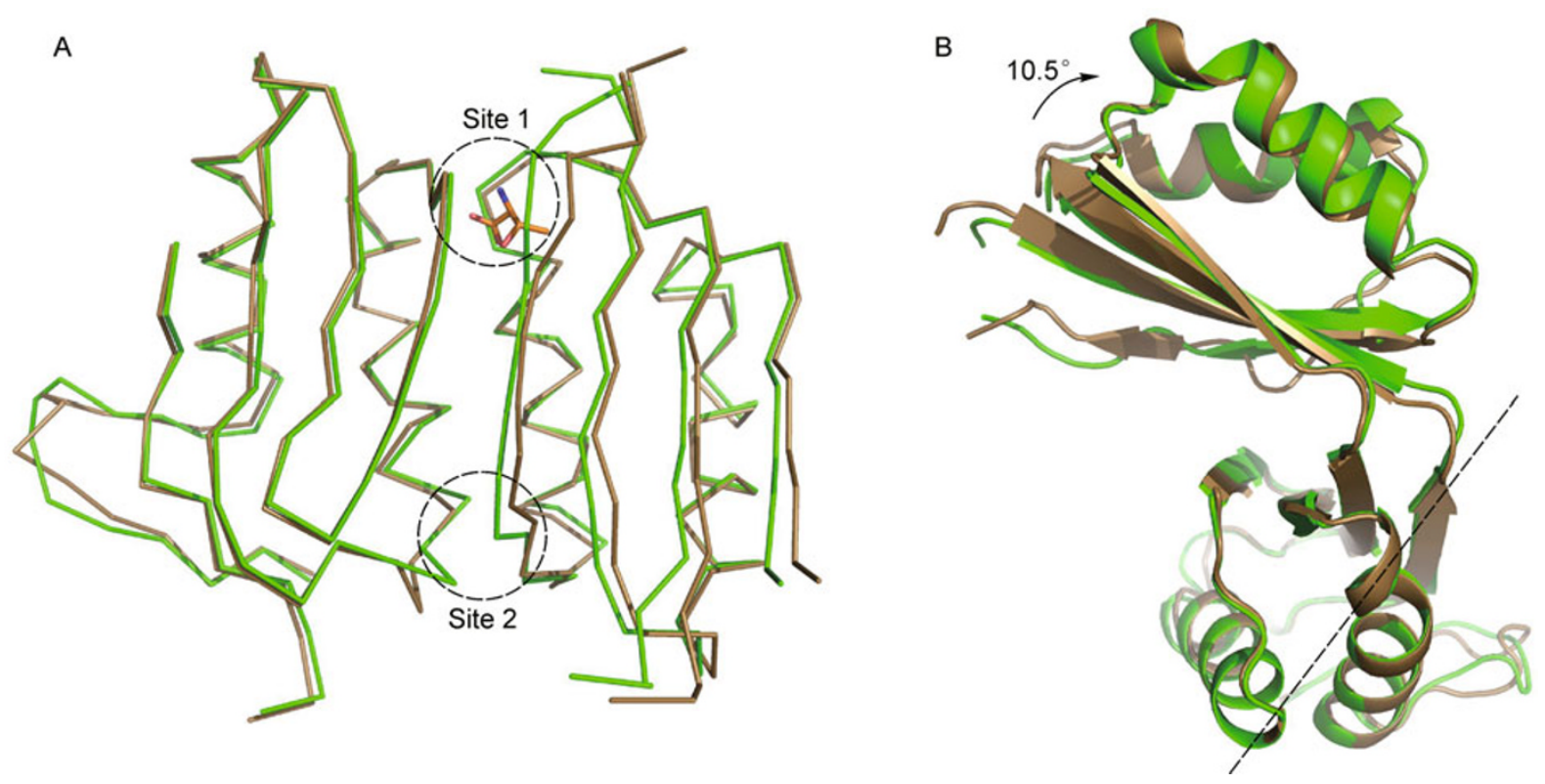

Figure 5. Comparison of a single inhibitor-binding unit between unliganded MtbAK $\beta$ and MtbAK $\beta$-Thr. (A) Superposition of the inhibitor-binding units of unliganded MtbAK $\beta$ and MtbAK $\beta$-Thr. MtbAK $\beta$-Thr and unliganded MtbAK $\beta$ are in green and sand, respectively. The Thr molecule is depicted as a stick model. ACT1 shows the Can models of the $Y$ chain (residues 1-11 and 95-168) in MtbAK $\beta$-Thr and unliganded MtbAK $\beta$, and ACT2 shows Ca models of the $X$ chain (residues 12-94) in MtbAK $\beta$-Thr and unliganded MtbAK $\beta$. (B) Domain motion in MtbAK $\beta$ caused by Thr binding. The structures of MtbAK $\beta$-Thr and unliganded MtbAK $\beta$ are shown in green and sand, respectively. Domain motion was resolved using DYNDOM (Hayward and Lee, 2002). A dash line demonstrates hinge axis of the movement.

Table 2 Analysis of the dimer interface by the Protein-Protein Interaction Server (http://www.biochem.ucl.ac.uk/bsm/PP/ server)

\begin{tabular}{lcc}
\hline Interface parameters & MtbAK $\beta$-free & MtbAK $\beta$-Thr \\
\hline $\begin{array}{l}\text { Interface accessible } \\
\text { surface area }\left(\AA^{2}\right)\end{array}$ & 1814 & 2065 \\
$\begin{array}{l}\text { \% of monomer accessible } \\
\text { surface area }\end{array}$ & 20.99 & 23.86 \\
\% Polar residues in interface & 24.49 & 24.07 \\
\% Non-polar residues in & 51.02 & 50.00 \\
$\begin{array}{l}\text { interface } \\
\% \text { Charged residues in }\end{array}$ & 24.49 & 25.93 \\
$\begin{array}{l}\text { interface } \\
\text { Hydrogen bonds }\end{array}$ & 6 & 22 \\
Salt bridges & 26 & 62 \\
\hline
\end{tabular}

MtbAK $\beta$-Thr is similar to that of $\mathrm{CgAK} \beta$ monomer bound by a threonine. The rmsd for Ca between these two structures is $0.9 \AA$. Threonine is stabilized by ionic bonds between the $\mathrm{Asp}^{274(25)}-\mathrm{O}^{\Omega 2}$ and the amino group of threonine, and the $\mathrm{GIn}^{298(49)}-\mathrm{N}^{\varepsilon 2}$ and the hydroxyl group of threonine. The amino group of threonine is stabilized by lle126 O and Asn125- $\mathrm{O}^{\Omega 1}$ via hydrogen bonds, while the carboxyl group of threonine forms a hydrogen bond with lle126 N. The methyl group of threonine is recognized by hydrophobic interactions with Thr59 and lle61. Two water molecules around two oxygen atoms of the carboxyl group of threonine are involved in a hydrogen-bond network between the carboxyl group of threonine, Ala30 N, Ala31 N, Gly28 N, and Val124 O. Therefore, the carboxyl group of Thr is stabilized by positively charged main chain atoms. As observed in CgAK $\beta$ and TtAK $\beta$, the carboxyl group of Thr is located near the $\operatorname{Pro}^{27}(\beta)$ $\operatorname{Gly}^{28}(\beta)$ sequence at the $N$ terminus of the $\beta$ subunit, implying that the positive charges of the helix dipole participate in the recognition of the carboxyl group. Thus, Thr is recognized by several ionic bonds, hydrogen bonds, and hydrophobic interactions. Most residues interacting with threonine in MtbAK $\beta$ are conserved in CgAK $\beta$ and TtAK $\beta$ and these residues may constitute a conserved inhibitory binding pocket in $\alpha_{2} \beta_{2}$-type AKs, which might share similar regulatory mechanisms.

Enzymatic assays in our study have shown that MtbAK is significantly inhibited by the addition of threonine but not lysine (Fig. 4C). Thus, threonine may be important for the regulation of MtbAK enzymatic activity.

\section{Conformational change of MtbAK $\beta$ upon Thr binding and its implications in the regulatory mechanism}

One difference between MtbAK $\beta$-Thr and MtbAK $\beta$-free is that 
the $\beta$ strands around the Thr-binding site exhibit outward shifts in the presence of $\mathrm{Thr}$, with $10.5^{\circ}$ rotation of the ACT2 domain from the ACT1 domain (Fig. 5B). MtbAK and CgAK are both heterotetramer with an $\alpha_{2} \beta_{2}$ configuration. The structure of MtbAK $\beta$ monomer shares high similarity with $\mathrm{CgAK} \beta$ monomer. The regulatory region of $\mathrm{CgAK}$ is composed of a dimer, and enzymatic activity of $\mathrm{CgAK}$ is inhibited by both Lys and Thr in a concerted manner. One threonine molecule is bound at site 1 in CgAK $\beta$, like MtbAK $\beta-T h r$ (Yoshida et al., 2007a). Thus, a similar conformational change is anticipated for the two enzymes upon the absence or presence of Thr and the regulatory mechanism of MtbAK is discussed.

In CgAK, mutations of residues in the $\beta 3-\beta 4$ loop which are close to site 1 made the enzyme insensitive to lysine (Yoshida et al., 2007a). Lys is bound to site 2 between ACT1 and ACT2, which is from a different chain (Yoshida et al., 2010 ). The influence of residues in the $\beta 3-\beta 4$ loop on the catalytic regulation of CgAK leads to the analysis of the $\beta 3-\beta 4$ loop of MtbAK. In MtbAK, outward shifts of $\beta 3-\beta 4$ triggered by Thr binding induce shifts surrounding site 2 . It is likely that a conformational change at site 2 triggered by Thr binding at site 1 will induce an additional Thr binding at site 2 in MtbAK. Additionally, in MtbAK, the Pro27- Gly28 sequence at the $N$ terminus of the $\beta$ subunit participates in the binding of Thr. The dihedral angle of Gly28 has a significant change upon Thr binding. In chain A of MtbAK-free, $\Phi=$ $107.47^{\circ}, \Psi=-39.64^{\circ}$, while in MtbAK-Thr, $\Phi=94.26^{\circ}, \Psi=$ $-10.1^{\circ}$ in chain A. The Pro27- Gly28 sequence is conserved in CgAK for the recognition of Thr. The equivalent Pro-Gly motif Pro109- Gly110 surrounding site 2 in $\operatorname{CgAK} \beta$ is conserved in MtbAK $\beta$, and the dihedral angle of Gly110 also changes upon Lys binding at site 2 . In chain $\mathrm{O}$ of Thrbound CgAK, $\Phi=85.81^{\circ}, \Psi=-5.77^{\circ}$, while $\Phi=89.92^{\circ}, \Psi=$ $-0.75^{\circ}$ in chain $\mathrm{O}$ of $\mathrm{CgAK}$, upon Thr and Lys binding. According to the allowed or generously allowed regions on the Ramachandran plot, it is likely that a dramatic change can occur in the dihedral angle of Gly110 in MtbAK $\beta$. Thus, we propose that the Pro-Gly sequence is important for the induction of conformational change upon inhibitor binding in $\alpha_{2} \beta_{2}$-type AKs. However, more studies are needed to confirm whether an additional Thr is bound at site 2 and inhibits MtbAK.

There are structural differences between MtbAK $\beta$ and CgAK $\beta$. An additional $\beta$ strand $\beta 9$ was found at the $C$ terminus in $\operatorname{CgAK} \beta$. This $\beta$ strand is proposed to participate in the inhibition induced by Thr and Lys in CgAK. After Thr and Lys binding, $\beta 1(\beta)$ shifts from $\beta 9(\beta)$ to $\beta 5(\alpha)$, facilitating the adoption of an inactive closed conformation (Yoshida et al., 2010). However, MtbAK $\beta$ does not have this additional $\beta$ strand. In addition, the disordered residues in the $\beta 3-\beta 4$ loop region in MtbAK $\beta$ indicate that this is a flexible region. These may cause the differences in the regulatory mechanisms between MtbAK and CgAK.

\section{CONCLUSIONS}

Aspartate kinase from Mtb catalyzes the biosynthesis of the aspartate family amino acids, including lysine, threonine, isoleucine, and methionine. Since AK is critical for the survival of $M t b$ and does not exist in humans, it is an important target for anti-TB drugs.

We reported the crystal structures of the MtbAK-free and MtbAK-Thr at high resolutions. The structure of MtbAK $\beta$, composed of four-stranded $\beta$-sheets and two $\alpha$-helices on both sides respectively, contains conserved ACT domains and shares high similarity to $\operatorname{CgAK} \beta$ and TtAK $\beta$. Enzymatic assays showed that Thr binding inhibits MtbAK activity. Most residues involved in Thr binding are conserved in CgAK $\beta$ and TtAK $\beta$. We propose that these residues form an inhibitory binding pocket in $\alpha_{2} \beta_{2}$-type AKs and similar regulatory mechanisms might be shared by AKs from different species. According to the structure comparison of MtbAK-free and MtbAK-Thr, we analyzed the regulatory mechanism of MtbAK and speculated that Thr binding at site 1 may facilitate the binding of an additional Thr molecule at site 2 and inhibit the enzyme. The further elucidation of the regulatory mechanism of MtbAK relies on the crystal structure of $\alpha_{2} \beta_{2}$-form MtbAK in complex with Thr. The crystal structures of MtbAK $\beta$ and MtbAK-Thr we reported here provide useful information for drug design targeting MtbAK. Threonine analogs and other small molecules, which have high affinity for MtbAK, may induce similar conformational changes and enzymatic activity inhibition. These potential drugs may constitute an efficacious treatment for MDR and TB/HIV co-infection cases.

\section{MATERIALS AND METHODS}

\section{Cloning}

The $\beta$ subunit of aspartate kinase gene was amplified by PCR and inserted into the expression vector pET-28a (Novagen) using Ndel and $\mathrm{Xhol}$ sites. The resultant MtbAK $\beta$ protein contains a $\mathrm{His}_{6}$ tag at the $C$ terminus. The genes encoding the $\alpha$ and $\beta$ subunits of MtbAK are cloned into the $\mathrm{pET}$-26b expression vector (Novagen) between Ndel/EcoRI sites, and EcoRI/Xhol sites led by a typical ribosome binding site respectively. The resulting $\beta$ subunit contains a $\mathrm{His}_{6}$ tag at the $\mathrm{C}$ terminus.

\section{Expression and purification}

The recombinant $\mathrm{pET}$-28a plasmid was transformed into $E$. coli strain Transetta (DE3) Chemically Competent Cells (TransGen Biotech). The cells were grown in LB broth containing $50 \mathrm{mg} / \mathrm{L}$ kanamycin at $303 \mathrm{~K}$. When the OD600 of the culture reached $0.6,0.1 \mathrm{mmol} / \mathrm{L}$ isopropyl $\beta$-D-thiogalactopyranoside (IPTG) was added to induce gene expression for $12 \mathrm{~h}$. The cells were then harvested by centrifugation. The pellets were lysed in buffer A $(20 \mathrm{mmol} / \mathrm{L}$ Tris$\mathrm{HCl} \mathrm{pH} 7.5$ and $150 \mathrm{mmol} / \mathrm{L} \mathrm{NaCl}$ ) and subsequently disrupted by high pressure cell cracker. Cell debris was removed by centrifugation 
at $14,000 \mathrm{rpm}$ for $45 \mathrm{~min}$ at $277 \mathrm{~K}$. MtbAK $\beta$ was purified by Ni-NTA affinity chromatography and the protein was eluted by buffer $A$ supplemented with $300 \mathrm{mmol} / \mathrm{L}$ imidazole and subsequently subjected to gel-filtration chromatography. The purity of target protein was confirmed to be more than $92 \%$ by SDS-PAGE and coommassie blue stain. The protein was concentrated to $7.5 \mathrm{mg} / \mathrm{mL}$ and stored at $193 \mathrm{~K}$. The expression and purification of MtbAK in the $\alpha_{2} \beta_{2}$ form was identical to MtbAK $\beta$.

\section{Crystallization}

Crystallization of the $\beta$ subunit of $M$. tuberculosis aspartokinase was conducted at $290 \mathrm{~K}$ using the hanging-drop vapor-diffusion technique. The purified protein was in buffer $A$ for crystallization. Crystals were formed by mixing $1 \mu \mathrm{L}$ of the MtbAK $\beta$ solution with $1 \mu \mathrm{L}$ of reservoir solution. Small crystals appeared after 2 days of growth in $0.1 \mathrm{~mol} / \mathrm{L}$ Tris $\mathrm{pH} 8.5$ and $2.0 \mathrm{~mol} / \mathrm{L}$ ammonium phosphate. Wellshaped crystals having a final size of $30 \mu \mathrm{m} \times 30 \mu \mathrm{m} \times 60 \mu \mathrm{m}$ were obtained after optimization. Good crystals which diffract to $2.6 \AA$ were found in crystallization buffer containing $0.1 \mathrm{~mol} / \mathrm{L}$ Tris $\mathrm{pH} 8.5$ and $1.8 \mathrm{~mol} / \mathrm{L}$ ammonium phosphate. $10 \mathrm{mmol} / \mathrm{L} \mathrm{Thr}$ was added to buffer A for the crystallization of MtbAK $\beta$-Thr. Well-shaped crystals of MtbAK $\beta$-Thr having a final size of $50 \mu \mathrm{m} \times 50 \mu \mathrm{m} \times 100 \mu \mathrm{m}$ were obtained in the same reservoir solution and diffracted to $2.0 \AA$. The crystals were transferred into cryoprotectant solution containing synthetic mother solution and $25 \%(\mathrm{~V} / \mathrm{V})$ glycol. Then the crystals were flash-cooled in liquid nitrogen for the collection of $\mathrm{X}$-ray diffraction data.

\section{X-ray data collection, processing and structure determination}

The crystal of MtbAK $\beta$ diffracts to $2.6 \AA$ at $100 \mathrm{~K}$ using an MAR 165 CCD detector with $1.0000 \AA$ wavelength on beamline $1 \mathrm{~W} 2$ at Beijing Synchrotron Radiation Facility (BSRF). The HKL2000 package (Otwinowski and Minor, 1997) was used for data processing, integrating and scaling. The crystals of MtbAK $\beta$ belong to the 141 space group with unit cell parameters of $a=b=64.8 \AA, c=137.0 \AA$, $\alpha$ $=\beta=\gamma=90^{\circ}$. The crystal contains one molecule per asymmetric unit with a Matthews coefficient of $3.7 \AA^{3} / \mathrm{Da}$, corresponding to $67 \%$ solvent content (Bailey, 1994). The PHASER program (McCoy et al., 2007) was used to perform molecular replacement using the crystal structure of $\beta$ subunit of $C$. glutamicum (PDB code: 2DTJ) (Yoshida et al., 2007a) as a template. COOT (Emsley and Cowtan, 2004) and REFMAC were used for subsequent manual model building and structure refinement.

The crystal of MtbAK $\beta$-Thr diffracted to $2.0 \AA$ resolution at $100 \mathrm{~K}$ using an ADSC Q315 CCD detector with $0.9800 \AA$ wavelength in BL17A (Photon Facoty, Japan). The HKL2000 package (Otwinowski and Minor, 1997) was used for data processing, integrating and scaling. The crystals of MtbAK $\beta$-Thr belong to the 141 space group with unit cell parameters of $a=b=62.4 \AA, c=137.3 \AA, \alpha=\beta=\gamma=90^{\circ}$. The crystal contains one molecule per asymmetric unit with a Matthews coefficient of $3.3 \AA^{3} / \mathrm{Da}$, corresponding to $63 \%$ solvent content (Bailey, 1994). The PHASER program (McCoy et al., 2007) was used to perform molecular replacement to find the correct solution using the crystal structure of MtbAK $\beta$ as a template. COOT (Emsley and Cowtan, 2004) and PHINEX (Adams et al., 2002) were used for subsequent manual model building and structure refinement. We summarized the final refinement statistics in Table 1 and the structural figures were drawn using PyMOL (DeLano and Lam, 2005).

\section{Binding assay of the $\alpha$ subunits and $\beta$ subunits from MtbAK}

For the binding assay of the $\alpha$ subunits and $\beta$ subunits from MtbAK, the two proteins were expressed simultaneously and purified as above. About $2 \mu \mathrm{g}$ of the $\alpha$ subunits and $1 \mu \mathrm{g}$ of His-tagged MtbAK $\beta$ were added to the column having $300 \mu \mathrm{L}$ of $30 \%$ Ni-NTA slurry with the bottom outlet capped at $4^{\circ} \mathrm{C}$ for $1.5 \mathrm{~h}$. Then the cap was removed and the Ni-NTA resin-coupled proteins were washed with buffer A containing $150 \mathrm{mmol} / \mathrm{L}$ imidazole 6 times. The proteins were eluted with buffer A containing $150 \mathrm{mmol} / \mathrm{L}$ imidazole. Eluted proteins were analyzed by $15 \%$ SDS-PAGE and coommassie blue stain.

\section{Enzymatic assay}

The activity of MtbAK was assayed by the method used by Black and Wright (Black and Wright, 1955). The $200 \mu \mathrm{L}$ reaction mixture contained $200 \mathrm{mmol} / \mathrm{L}$ Tris- $\mathrm{HCl}, \mathrm{pH} 7.5,10 \mathrm{mmol} / \mathrm{L} \mathrm{MgCl}_{2}$, $500 \mathrm{mmol} / \mathrm{L}$ ammonium sulfate, $10 \mathrm{mmol} / \mathrm{L}$ potassium aspartate, $10 \mathrm{mmol} / \mathrm{L}$ ATP, $10 \mathrm{mmol} / \mathrm{L}$ threonine or lysine, $160 \mathrm{mmol} / \mathrm{L} \mathrm{NH} \mathrm{NH}_{2} \mathrm{OH}$ $\mathrm{HCl}$ (neutralized with $\mathrm{KOH}$ ), and enzyme at appropriate concentrations. After incubation at $30^{\circ} \mathrm{C}$ for $30 \mathrm{~min}$, the reaction was stopped by adding $300 \mu \mathrm{L} 5 \%(w / v) \mathrm{FeCl}_{3}$ solution, and absorbance was monitored at $540 \mathrm{~nm}$.

\section{ACKNOWLEDGEMENTS}

We thank staffs from Beijing Synchrotron Radiation Facility for their technical help with the data collection and Lei Wei for his assistance in manuscript revision. This work was supported by the National Basic Research Program (973 Program) (Grant Nos. 2011CB915501 and 2011CB910304) and National Major Project (Grant Nos. 2009ZX10004-304 and 2009ZX10004-802).

\section{ABBREVIATIONS}

AK, aspartate kinase; CgAK, aspartate kinase from Corynebacterium glutamicum; $\mathrm{CgAK} \beta$, the regulatory subunit of aspartate kinase from Corynebacterium glutamicum; Mtb, Mycobacterium tuberculosis; MtbAK, aspartate kinase from Mycobacterium tuberculosis; MtbAK $\beta$, the regulatory subunit of aspartate kinase from Mycobacterium tuberculosis; TtAK, aspartate kinase from Thermus thermophilus; TtAK $\beta$, the regulatory subunit of aspartate kinase from Thermus thermophilus; TyrA, prephenate dehydrogenase

\section{REFERENCES}

Adams, P.D., Grosse-Kunstleve, R.W., Hung, L.W., loerger, T.R., McCoy, A.J., Moriarty, N.W., Read, R.J., Sacchettini, J.C., Sauter, N.K., and Terwilliger, T.C. (2002). PHENIX: building new software for automated crystallographic structure determination. Acta Crystallogr D Biol Crystallogr 58, 1948-1954.

Aravind, L., and Koonin, E.V. (1999). Gleaning non-trivial structural, functional and evolutionary information about proteins by iterative database searches. J Mol Biol 287, 1023-1040.

Bailey, S., and the Collaborative Computational Project, Number 4. (1994). The CCP4 suite: programs for protein crystallography. Acta 
Crystallogr D Biol Crystallogr 50, 760-763.

Black, S., and Wright, N.G. (1955). beta-Aspartokinase and betaaspartyl phosphate. J Biol Chem 213, 27-38.

Chaitanya, M., Babajan, B., Anuradha, C.M., Naveen, M., Rajasekhar, C., Madhusudana, P., and Kumar, C.S. (2010). Exploring the molecular basis for selective binding of Mycobacterium tuberculosis Asp kinase toward its natural substrates and feedback inhibitors: a docking and molecular dynamics study. J Mol Model 16, 1357-1367.

Chan, E.D., and Iseman, M.D. (2008). Multidrug-resistant and extensively drug-resistant tuberculosis: a review. Curr Opin Infect Dis $21,587-595$.

Chipman, D.M., and Shaanan, B. (2001). The ACT domain family. Curr Opin Struct Biol 11, 694-700.

Cirillo, J.D., Weisbrod, T.R., Pascopella, L., Bloom, B.R., and Jacobs, W.R. Jr. (1994). Isolation and characterization of the aspartokinase and aspartate semialdehyde dehydrogenase operon from mycobacteria. Mol Microbiol 11, 629-639.

Cole, S.T., Brosch, R., Parkhill, J., Garnier, T., Churcher, C., Harris, D., Gordon, S.V., Eiglmeier, K., Gas, S., Barry, C.E., et al. (1998). Deciphering the biology of Mycobacterium tuberculosis from the complete genome sequence. Nature 393, 537-544. Nature 396, 190-198 (Erratum).

Corbett, E.L., Watt, C.J., Walker, N., Maher, D., Williams, B.G., Raviglione, M.C., and Dye, C. (2003). The growing burden of tuberculosis: global trends and interactions with the HIV epidemic. Arch Intern Med 163, 1009-1021.

DeLano, W.L., and Lam, J.W. (2005). PyMOL: A communications tool for computational models. Abstracts of Papers of the American Chemical Society 230, U1371-U1372.

Emsley, P., and Cowtan, K. (2004). Coot: model-building tools for molecular graphics. Acta Crystallogr D Biol Crystallogr 60, 2126-2132.

Faehnle, C.R., Liu, X., Pavlovsky, A., and Viola, R.E. (2006). The initial step in the archaeal aspartate biosynthetic pathway catalyzed by a monofunctional aspartokinase. Acta Crystallogr Sect F Struct Biol Cryst Commun 62, 962-966.

Fleischmann, R.D., Alland, D., Eisen, J.A., Carpenter, L., White, O., Peterson, J., DeBoy, R., Dodson, R., Gwinn, M., Haft, D., et al. (2002). Whole-genome comparison of Mycobacterium tuberculosis clinical and laboratory strains. J Bacteriol 184, 5479-5490.

Gilker J.M., and Jucker M.T. (1997). Mycobacterium tuberculosis askalpha, ask-beta and asd genes. Submitted (FEB-1997) to the EMBL/GenBank/DDBJ databases.

Grant, G.A. (2006). The ACT domain: a small molecule binding domain and its role as a common regulatory element. J Biol Chem 281, 33825-33829.

Hayward, S., and Lee, R.A. (2002). Improvements in the analysis of domain motions in proteins from conformational change: DynDom version 1.50. J Mol Graph Model 21, 181-183.

Kotaka, M., Ren, J., Lockyer, M., Hawkins, A.R., and Stammers, D.K. (2006). Structures of R- and T-state Escherichia coli aspartokinase
III. Mechanisms of the allosteric transition and inhibition by lysine. $\mathrm{J}$ Biol Chem 281, 31544-31552.

Mas-Droux, C., Curien, G., Robert-Genthon, M., Laurencin, M., Ferrer, J.L., and Dumas, R. (2006). A novel organization of ACT domains in allosteric enzymes revealed by the crystal structure of Arabidopsis aspartate kinase. Plant Cell 18, 1681-1692.

Matthews, B.W. (1968). Solvent content of protein crystals. J Mol Biol 33, 491-497.

McCoy, A.J., Grosse-Kunstleve, R.W., Adams, P.D., Winn, M.D., Storoni, L.C., and Read, R.J. (2007). Phaser crystallographic software. J Appl Crystallogr 40, 658-674.

Nishiyama, M., Kukimoto, M., Beppu, T., and Horinouchi, S. (1995). An operon encoding aspartokinase and purine phosphoribosyltransferase in Thermus flavus. Microbiology 141, 1211-1219.

Otwinowski, Z., and Minor, W. (1997). Processing of X-ray diffraction data collected in oscillation mode. Macromolecular Crystallography . Pt A 276, 307-326.

Rapaport, E., Levina, A., Metelev, V., and Zamecnik, P.C. (1996). Antimycobacterial activities of antisense oligodeoxynucleotide phosphorothioates in drug-resistant strains. Proc Natl Acad Sci U S A 93, 709-713.

Robin, A.Y., Cobessi, D., Curien, G., Robert-Genthon, M., Ferrer, J.L., and Dumas, R. (2010). A new mode of dimerization of allosteric enzymes with ACT domains revealed by the crystal structure of the aspartate kinase from Cyanobacteria. J Mol Biol 399, 283293.

Rognes, S.E., Lea, P.J., and Miflin, B.J. (1980). S-adenosylmethionine-a novel regulator of aspartate kinase. Nature 287, 357-359.

Schuldt, L., Suchowersky, R., Veith, K., Mueller-Dieckmann, J., and Weiss, M.S. (2011). Cloning, expression, purification, crystallization and preliminary $\mathrm{X}$-ray diffraction analysis of the regulatory domain of aspartokinase (Rv3709c) from Mycobacterium tuberculosis. Acta Crystallogr Sect F Struct Biol Cryst Commun 67, 380-385.

Tomioka, H., and Namba, K. (2006). [Development of antituberculous drugs: current status and future prospects] . Kekkaku 81, 753-774.

Yoshida, A., Tomita, T., Kono, H., Fushinobu, S., Kuzuyama, T., and Nishiyama, M. (2009). Crystal structures of the regulatory subunit of Thr-sensitive aspartate kinase from Thermus thermophilus. FEBS J 276, 3124-3136.

Yoshida, A., Tomita, T., Kurihara, T., Fushinobu, S., Kuzuyama, T., and Nishiyama, M. (2007a). Structural Insight into concerted inhibition of alpha 2 beta 2-type aspartate kinase from Corynebacterium glutamicum. J Mol Biol 368, 521-536.

Yoshida, A., Tomita, T., Kuzuyama, T., and Nishiyama, M. (2007b). Purification, crystallization and preliminary X-ray analysis of the regulatory subunit of aspartate kinase from Thermus thermophilus. Acta Crystallogr Sect F Struct Biol Cryst Commun 63, 96-98.

Yoshida, A., Tomita, T., Kuzuyama, T., and Nishiyama, M. (2010). Mechanism of concerted inhibition of alpha2beta2-type heterooligomeric aspartate kinase from Corynebacterium glutamicum. J Biol Chem 285, 27477-27486. 